\title{
Strategieoptionen für Unterhaltungsproduzenten in Europa
}

Baumgartner, Daniel ; Moutragi, Diana ; Tomic, Matej ; von Rimscha, M Bjørn

Posted at the Zurich Open Repository and Archive, University of Zurich

ZORA URL: https://doi.org/10.5167/uzh-54285

Book Section

Published Version

Originally published at:

Baumgartner, Daniel; Moutragi, Diana; Tomic, Matej; von Rimscha, M Bjørn (2012). Strategieoptionen für Unterhaltungsproduzenten in Europa. In: Müller-Lietzkow, Jörg. Ökonomie, Qualität und Mangement von Unterhaltungsmedien. Baden-Baden: Nomos, 201-223. 


\title{
Strategieoptionen für Unterhaltungsproduzenten in Europa
}

\author{
Daniel Baumgartner, Diana Moutragi, Matej Tomic und M. Bjorn von Rimscha
}

\section{Wandel in der Produktionslandschaft}

Über nationale Medienmärkte und unterschiedliche Gattungen und Genres hinweg sehen sich Medienunternehmen weitreichenden Veränderungen innerhalb und außerhalb der Organisation ausgesetzt. Produktionsmethoden ändern sich, trotz Konzentrationstendenzen nimmt der Wettbewerb zu, die Nachfrage von Rezipienten und Werbetreibenden verändert sich und wo Wertschöpfungsketten umstrukturiert werden, ändern sich auch die Machtkonstellationen zwischen den beteiligten Akteuren. Veränderungen auf der Strukturebene sollten Anpassungen des strategischen Verhaltens nach sich ziehen. Dieser Beitrag fokussiert auf den Markt für audiovisuelle Unterhaltung und konkret auf die Produktionsfirma als inhaltlichen und technischen Produzenten der Inhalte.

Die aktuelle Herausforderung für Medienunternehmen besteht in der Kombination aus steigender Komplexität des Marktes und einer ebenfalls steigenden Unsicherheit über die Marktentwicklung (Picard 2004: 3). Steigende Komplexität lässt sich z.B. an der wachsenden Zahl von Marktsegmenten und somit potenziellen Konkurrenten ablesen, aber auch an der anhaltenden Tendenz zu Cross Media Strategien, wobei Medienunternehmen in mehreren Märkten gleichzeitig operieren. Marktunsicherheit zeigt sich in der Volatilität von Märkten und der Ungewissheit über die Ergebnisse des eigenen Handelns. Marktunsicherheit verlangt erhöhte Innovationsbereitschaft und somit ein riskanteres Verhalten. Die Schwierigkeit für Medienunternehmen liegt in der Widersprüchlichkeit der adäquaten Reaktion auf steigende Komplexität einerseits und steigende Unsicherheit andererseits. Komplexe Märkte verlangen eine strikte Kontrolle über Strategie und Produktion, unsichere Märkte hingegen verlangen Flexibilität.

Die Produktion von audiovisueller Unterhaltung erfolgt im Gegensatz zum Packaging und zum Vertrieb in der Regel in kleinen Unternehmen. Selbst in den Fällen, in denen Produktionsfirmen Teil eines integrierten Konzerns sind, ist eine wertschöpfungsstufenübergreifende Strategie eher unüblich. RTL kauft Unterhaltungsprogramme durchaus nicht nur 
bei Produzenten der FremantleMedia (Fröhlich 2010). Obschon der Anpassungsdruck hoch ist, diagnostiziert Picard Defizite, die eine strategische Reaktion erschweren: „Media managers' knowledge of strategy and strategy processes are limited and most media firms do not have strategy units or organized business intelligence activities" (Picard 2004: 2). Für Kleinbetriebe in der Produktion muss dies umso mehr gelten. Im Gegensatz zur Nachrichtenproduktion spielen in der Unterhaltung normative Ziele eine geringere Rolle (Altmeppen 2008), sodass sich trotz der genannten Defizite dieser Bereich besonders für eine Untersuchung des ökonomisch induzierten, strategischen Verhaltens eignet. In der Unterhaltungsproduktion ist der ökonomische Imperativ ausgeprägter als in anderen Marktsegmenten. Gleichzeitig ist der Spielraum für diese Akteure, die jeweils in zeitlich befristete Projektnetzwerke eingebunden sind, begrenzt (Sydow/ Windeler 2004: 41). Ihre Abhängigkeit von Distributoren ist groß (Lantzsch 2008: 95-100; von Rimscha: 2008) und entsprechend groß könnte die Motivation sein, Veränderungen der Rahmenbedingungen zu nutzen, um die eigene Marktmacht zu stärken. Im Folgenden soll anhand einer empirischen Studie mit Produktionsfirmen in Europa dargestellt werden, wie das Produzentenverhalten angepasst werden könnte oder bereits angepasst wird, um die Probleme unter sich ändernden Bedingungen zu überwinden und um sich eine strategisch vorteilhafte Position zu verschaffen.

\section{Zum Stand der Forschung}

\subsection{Strategie unter dynamischen Marktbedingungen}

Wirtz zeigt für das strategische Management im Medienbereich mehrere Möglichkeiten der beteiligten Unternehmen auf (Wirtz 2009: 321ff.). Zum einen nennt er die Fokussierungs- und Netzwerkstrategie, die dem besonderen Charakter von audiovisuellen Produkten entspricht. Im TVBereich sind die Marktteilnehmer meist klar spezialisiert. Kaum ein TVSender produziert, jenseits von Nachrichten und Informationsmagazinen, seine Inhalte selbst. Versuche von Kabelnetzbetreibern oder Telekommunikationsfirmen im Contentgeschäft $\mathrm{Fuß}$ zu fassen, sind in der Regel gescheitert: Die Schweizer Cablecom z. B. hat Pläne ihren Infokanal zum echten TV-Sender auszubauen aufgegeben. Umgekehrt verkaufen Produktionsfirmen ihre Inhalte meist nicht direkt an die Rezipienten, sondern überlassen das Marketing im Endkundengeschäft den Sendern. 
Bei Betrachtung der Wertschöpfungsstrukturen im TV-Unterhaltungsbereich ist festzustellen, dass die Inhalte von der Beschaffung der Inputfaktoren über die Programmproduktion, den Rechtehandel, das Packaging bis zur Distribution einen langen Weg zurücklegen, um schließlich die Zuschauer erreichen zu können. Produktionsunternehmen sind dabei einer von vielen Akteuren, denen in der Wertschöpfungskette jeweils begrenzte Aufgaben zugeordnet sind. Die Produktion von Unterhaltungsinhalten beginnt mit einer Idee, die beim Sender, dem Produktionsunternehmen oder auch auf weiter vorgelagerten Stufen, wie etwa einen unabhängigen Autor, entsteht und nach bestimmten Auswahlprozessen im besten Fall zu einem Drehbuch entwickelt wird. Dieses wird anschließend in der eigentlichen Produktion technisch umgesetzt. In der Postproduktion wird das erstellte Material, heute meist in digitaler Form, geschnitten, korrigiert und vertont, bevor es schließlich ausgestrahlt werden kann. Neben den Produktionsressourcen (Studios, Ausstattung) nennt Wirtz (2009: 409) vor allem die personellen Ressourcen, die im Produktionsprozess eine hohe Relevanz haben: Autoren, Schauspieler, Regisseure, Moderatoren, sowie technische Mitarbeiter der Produktion und Postproduktion. Im Zuge der Entwicklung vom Producer TV zum Publisher TV (Tunstall 1993) werden die Inhalte in der Unterhaltungsproduktion längst nicht mehr in starren, isolierten Organisationen realisiert, sondern in fluiden, zeitlich befristeten Projektnetzwerken. Obwohl die Unternehmen formal unabhängig sind, findet der Austausch nicht immer über den Markt statt. Hohe Such- und Transaktionskosten machen wiederholte Zusammenarbeit in Netzwerken attraktiv. Sydow und Windeler bezeichnen diese als „Organisationsform ökonomischer Aktivitäten zwischen rechtlich selbstständigen, wirtschaftlich mehr oder weniger abbängigen Unternebmungen zur Abwicklung zeitlich befristeter Aufgaben, wobei sich die Koordination der Projekte [...] im rekursiven Zusammenspiel projektbezogener und projek.tübergreifender Aktivitäten und Beziehungen zwischen den Netzwerkunternehmungen vollriebt" (Sydow/ Windeler 2004: 41). Dieses temporäre System wird von Produktionsunternehmen, gemeinsam mit den Sendern, organisiert und koordiniert. Die Verbindung zwischen den beiden Akteuren ist dabei meistens so eng, dass die jeweiligen internen Prozesse gegenseitig bekannt sind. Zwischen Produzent und Sender herrscht ein Spannungsverhältnis von Kooperation und Konkurrenz, das sich in der Ausgestaltung der Produktionsprozesse und der Gewinn- und Rechteverteilung manifestiert (Sydow/ Windeler 2004: 43-44). Wenn hohe First-Copy-Kosten marginalen Kosten der Wiederverwertung gegenüberstehen, stellt sich 
immer die Frage: „Who will reap the benefit?" (Doyle 2002: 81) Die Sender haben meist eine größere Verhandlungsmacht und können sich so die kompletten Programmrechte sichern, selbst wenn diese jenseits des eigenen Programms kaum verwertet werden. Im Zuge der Digitalisierung verschärft sich die Rechtefrage, da für mehr Distributionskanäle mehr Wiederholungen benötigt werden (Lantzsch 2008: 100). Diese Entwicklung könnte Produktionsunternehmen in eine bessere Verhandlungsposition bringen. Branded Entertainment kann in diesem Zusammenhang insofern eine Rolle spielen, als dass Produzenten sowohl durch die Etablierung der eigenen Distribution, als auch durch eine Stabilisierung der Finanzierungsflüsse den Machtasymmetrien entgegensteuern könnten.

Als zweite Basisstrategie nennt Wirtz die Integrationsstrategie, die es erlaubt wegfallende Erlöse aus klassischen Absatzmärkten (z. B. Kinokasse) auf anderen Verwertungsstufen auszugleichen (Wirtz 2009: 313). So besorgen Filmverleiher und TV-Sender den Vertrieb von DVDs mit ihren Inhalten meist über eigene Tochtergesellschaften. Für Unterhaltungsproduzenten, die die Rechte nicht komplett an Sender oder Verleiher verkauft haben, ließe sich dies beispielsweise mit einer eigenen Online-Plattform erreichen. Gleichermaßen lässt sich auch die Werbeakquisition in Produktionsfirmen integrieren, sodass die rückläufige Zahlungsbereitschaft der Sender kompensiert werden könnte.

Allgemein ist von Unternehmen in dynamischen Umfeldern innovatives Handeln gefordert, um bestehen zu können und erfolgreich zu bleiben. Miller und Friesen (1982) haben sich vor diesem Hintergrund mit der Innovationskraft von Unternehmen befasst und unterscheiden konservative und unternehmerisch denkende Firmen. Den beiden Typen liegen vier Bestimmungsfaktoren für die Innovationsbereitschaft zugrunde: das Marktumfeld, die Organisationsstruktur und die Art der Informationsverarbeitung und Entscheidungsfindung. Miller und Friesen verstehen Innovation dabei reaktiv, das heißt, als Voraussetzung für innovatives Handeln muss eine Veränderung im Umfeld des Unternehmens stattfinden. Je dynamischer die Branche ist, desto grösser ist die Notwendigkeit von Innovation. Um auf Veränderungen eingehen zu können, muss spezifisches Wissen vorhanden sein, das sich im Rahmen eines Monitoring aufbauen lässt. Weiter müssen Ressourcen, Fähigkeiten und strukturelle Hilfsmittel sowie Entscheidungsinstanzen vorhanden sein um innerhalb eines Unternehmens innovativ handeln zu können (Miller/ Friesen 1982: 3-6). In seiner Studie zu kleinen kreativen Unternehmen ver- 
wendet Chaston (2008) den Ansatz von Miller und Friesen um die unternehmerische Orientierung dieser Unternehmen zu bestimmen. Trotz des Strebens nach finanziellem Erfolg, schätzt Chaston die kleinen kreativen Unternehmen nicht als unternehmerisch denkend ein. Im Vordergrund steht der Konflikt zwischen Markt und Produkt also auch Fragen der ideologischen und künstlerischen Motivation. Chaston kann jedoch zeigen, dass die gering ausgeprägte unternehmerische Orientierung keinen Einfluss auf Leistungen des Unternehmens hat (Chaston 2008: 827). Das sich stetig wandelnde Umfeld von Lifestyle und Kunst scheint für kleine kreative Unternehmen Antrieb genug, sodass in diesem Fall eine unternehmerische Orientierung keine zwingende Voraussetzung für Innovationskraft darstellt.

\subsection{Strategien von Produktionsunternehmen}

Konkrete Studien zu Strategieoptionen von Produktionsfirmen sind rar. Rott und Zabel (2009) haben mögliche Strategien diskutiert und in einer Expertenbefragung mit deutschen Produzenten bewerten lassen. Sie gehen davon aus, dass technische Innovationen auf Basis der Digitalisierung und Datenkompression den Vertrieb von Inhalten über neue Kanäle ermöglichen. Heute lässt sich beobachten, wie allen voran die Sender ihre Inhalte vermehrt online anbieten. Für TV-Produktionsunternehmen stellt sich somit die Frage, wie im Zuge dieser Entwicklungen agiert werden soll, um auch weiterhin unternehmerisches Wachstum zu sichern. Für die entsprechende Analyse lässt sich die Produkt-Markt-Matrix heranziehen (Ansoff 1968), welche vier strategische Optionen für Unterhaltungsproduzenten offenlegt. Rott und Zabel greifen dieses Konzept auf und schlagen aufgrund der ,geringen Dynamik des bestebenden TV-Marktes und neu entstehender Märkte im Online-Bereich" die Marktentwicklung als naheliegende Wachstumsstrategie vor (Rott/ Zabel 2009: 94). Für Produktionsfirmen heißt das, dass neu auch Inhalte für Online-TV produziert werden sollen (vgl. auch Zabel 2008). Der Markteinstieg sei vor allem in jenen Bereichen attraktiv, in denen sich Online-Abnehmer durch aufwendige Inhalte von der Konkurrenz differenzieren wollen (Rott/ Zabel 2009: 101). In dieser sogenannten Internet-Publizistik gewinnen Querverweise auf andere Onlineinhalte und die Einbindung der Nutzer in die Zusammenstellung des Angebots an Bedeutung. Die Vorwärtsintegration der Distribution und den Aufbau von Kompetenz als Distributor mit eigenem Branding sehen Rott und Zabel zwar als Option, geben ihr aber eher geringe Umsetzungschancen. 
Przybylski (2010) sieht die Konvergenz von verschiedenen Distributionskanälen von audiovisuellen Inhalten bereits als vollzogen und spricht allgemein von einem Bewegtbildmarkt. In diesem sieht sie große Veränderungen in den Akteurskonstellationen, wobei Partner von heute in Zukunft zu Konkurrenten werden könnten. Das Internet löst den Fernseher von seiner Rolle als alleiniger Träger für audiovisuelle Unterhaltungsinhalte ab. Die klassischen Medien werden durch das Internet verzichtbar, da letzteres alle physischen Elemente für die Informationsvermittlung bereits mit sich bringt. Die klassischen Akteure müssen sich neu positionieren und aktiv neue Konzepte entwickeln, da bisherige Geschäftsmodelle durch tiefe Markteintrittsbarrieren verändert werden und neue Player aus anderen Branchen in den Markt eindringen. Die Innovationskraft der Unternehmen ist in einem solchen Umschwung gefordert, nur gute Qualität und starke Marken können sich in einem solchen Umfeld durchsetzten. Przybylski hat durch 16 Leitfadeninterviews mit deutschen Produktionsfirmen das Bewusstsein für den Wandel und die Stimmung in der Branche aufgezeigt. Die Produzenten versuchen sich vorsichtig auf das Internet auszurichten und ziehen es teilweise auch als eigene Vertriebsmöglichkeit in Betracht. Insgesamt möchten sie sich jedoch auf die Produktion als ihre Kernkompetenz konzentrieren, da bisher die notwendigen Ressourcen und das technische Know-how für den Eigenvertrieb fehlen. Ein gewisses Potenzial für neue Geschäftsfelder sehen sie in der Zusammenarbeit mit der Werbeindustrie und der Unternehmenskommunikation. Jedoch betrachten viele Produktionsfirmen die werbetreibende Wirtschaft noch nicht als Auftraggeber oder Partner, sondern sehen sie noch weitgehend in der Rolle des Käufers von Werbezeit. Auch Fröhlich verwendet Leitfadeninterviews mit Branchenvertretern um „Innovationssysteme der TV-Unterbaltungsproduktion“ (Fröhlich 2010) zu untersuchen. Neben Inhalten und der Technik sieht sie auch das Geschäftsmodell als ein Feld in dem Innovationen zum Erfolg führen können (Fröhlich 2010: 165ff.). Sowohl bei der Aufzeichnung und anderen Stufen des Herstellungsprozesses, als auch in der Distribution eröffnen sich neue Möglichkeiten durch die Digitalisierung und das Internet. In Bezug auf wirtschaftliche oder GeschäftsmodellInnovationen erwähnt Fröhlich lediglich den Formathandel (Fröhlich 2010). Dieser ist aus Perspektive der Produktionsfirmen als Mehrfachverwertung der Entwicklungskompetenz zu verstehen. Eine Vorwärtsintegration der Distribution oder die Werbefinanzierung der Produktion ist in ihren Interviews kein Thema gewesen. Fröhlich argumentiert, dass 
Innovationssysteme nicht isoliert und auf einzelne Akteure bezogen gesehen werden sollten: Neuheiten bei Produktionsunternehmen sind sowohl das Ergebnis von, als auch Anstoß für Innovationen bei vor- und nachgelagerten Akteuren (Fröhlich 2010: 421). Dabei gilt es nationale Unterschiede zu beachten, die sich aus unterschiedlichen Rahmenbedingungen und einem abweichenden Verständnis von der Notwendigkeit und Sinnhaftigkeit von Innovation speisen. Wo Fröhlich auf den Bereich der inhaltlichen Innovation fokussiert, soll das im Folgenden vorgestellte Projekt sich auf die wirtschaftlichen Strategien für die Produzenten konzentrieren.

\section{Forschungsfrage}

Die vorliegende Studie adressiert die Fragestellung, wie Veränderungen der technologischen, ökonomischen und regulativen Rahmenbedingungen das Handeln von Produktionsfirmen audiovisueller Inhalte beeinflussen.

Im Gegensatz zu den genannten Studien von Rott und Zabel (2009) und Przybylski (2010) soll die Frage dabei nicht mit Hilfe von Leitfadeninterviews beantwortet werden, sondern in einer quantitativen Befragung. Auch ist der Fokus nicht allein auf den deutschen Markt gerichtet, sondern auf verschiedene Märkte in Europa ausgeweitet, um den potenziellen Einfluss unterschiedlicher Rahmenbedingungen auf den nationalen Märkten erfassen zu können.

Die maßgeblichen Veränderungen sehen wir auf technologischer, ökonomischer und regulatorischer Ebene. Der technologische Aspekt ist geprägt durch die alles umfassende Digitalisierung, die in der ganzen Medienbranche Einzug hält. Für die audiovisuelle Unterhaltungsproduktion sind die Implikationen weitreichend: Für die Inhalte entstehen neue Darstellungs-, Speicherungs- und Distributionsmöglichkeiten, die Herstellung gestaltet sich dadurch schneller und günstiger als in analogen Produktionssystemen, und Mediennutzer werden durch multiple Kanäle in einer größeren Breite erreicht. Für die Produzenten ergeben sich neue Angebotsmöglichkeiten, für das Publikum neue Nutzungsformen. Die Entwicklung von mobilen, multimedialen Endgeräten und die Verbreitung von breitbandigen Internet- und Mobilzugängen sind typische Treiber. Für Produktionsunternehmen ist das insofern von Bedeutung, als dass TV Sender nicht mehr den einzig möglichen Verbreitungsweg für audiovisuelle Unterhaltung darstellen. Neue Vertriebskanäle vereinfachen den Markteintritt von neuen Distributoren oder ermöglichen die 
Vorwärtsintegration der Distribution durch Produzenten. Nicht außer Acht zu lassen sind auch die nutzungsseitigen Verschiebungen zugunsten des Internets, ein Trend, der sich in Zukunft noch verstärken dürfte (Wirtz 2009: 38). Für den Nutzer entstehen dadurch nicht nur mehr Programme, sondern durch die Interaktivität wahrscheinlich auch die attraktiveren. Wie sich die Nutzerpräferenzen entwickeln werden, welche Unterhaltungsbedürfnisse die Anbieter zu befriedigen haben und welche Rolle die Personalisierung und Individualisierung von Inhalten einnimmt, muss ebenfalls in die strategischen Entscheide von Produktionsfirmen einfließen.

Legt man den Fokus auf die ökonomische Ebene, so hat sich die von Tunstall (1993) diagnostizierte Tendenz zum ,publisher television" mittlerweile auch bei Public Service Sendern allgemein durchgesetzt. Die Sender haben sich aus der Produktion zurückgezogen und treten nur mehr als Auftraggeber und Packager auf. Im angelsächsischen Raum inzwischen eher die Ausnahme, ist mittlerweile auch in Deutschland der, Total Buy-Out' nicht mehr die einzig übliche Vertragsform zwischen Sender und Produzent. Sender wälzen zunehmend Entwicklungsrisiken auf Produzenten ab. Diese sind gezwungen sich durch die Verwertung der bei ihnen verbleibenden Rechte zu refinanzieren. Weiter ist dem Werbemarkt Beachtung zu schenken. Aktive Werbevermeidung (z. B. mittels entsprechenden DVRs oder beim catch up TV) wird im klassischen Fernsehen zunehmend problematisch, was aufseiten der Werbetreibenden hohe Ausgaben unattraktiv macht. Neue Formen, etwa im Bereich programmintegrierter Werbung, gewinnen so an Bedeutung.

$\mathrm{Zu}$ guter Letzt sind die Veränderungen der regulativen Rahmenbedingungen mit einzubeziehen, denn sie ermöglichen und begrenzen das Handeln von Produktionsfirmen fundamental. Aus der Tradition der Telekomregulierung sind netzbasierte Medien inhaltlich weniger reguliert. Doch auch im Rundfunkbereich lassen sich Deregulierungstendenzen beobachten, die die Akteursbeziehungen verändern, etwa in Bezug auf die Trennung von Werbung und Programm. Mit der Umsetzung der EU Richtlinie über audiovisuelle Mediendienste (AVMD-Richtlinie 2010/13/EU) sind seit April 2010 auch in Deutschland Product Placements in gewissen Grenzen zulässig. Wenn Werbebotschaften integrale Bestandteile des Programms sind, stellt sich die Frage, warum sie erst auf Ebene des Packagings und der Distribution eingefügt werden sollten. Auch im Zusammenhang mit cross-sektoralem Wettbewerb, Integrationsregeln und Eigentumsstrukturen hat es z. T. weitgehend Lockerun- 
gen gegeben. Hieraus ergeben sich Konzentrationstendenzen auch auf Ebene der Produktion (Pätzold/ Röper 2003: 26; Przybylski 2010: 54), die wiederum Einfluss auf die Strategieoptionen haben.

Zwei Beispiele zeigen mögliche Anpassung des Geschäftsmodells von Produktionsfirmen in Reaktion auf die skizzierten Veränderungen: Brainpool, eine der wenigen deutschen Produktionsfirmen, die offensiv einen eigenen Rechtestock aufbauen, ist seit 2008 mit der Internetmarke MySpass im Endkundengeschäft aktiv. Im selben Jahr gründete Endemol UK, eine der größten britischen Produktionsfirmen, die Unternehmenseinheit New State, die im Rahmen von Branded Entertainment die direkte Zusammenarbeit mit der Werbebranche forcieren soll. In ersten Fall wird Entertainment gebranded, im zweiten Entertainment für Brands geöffnet. Diese zwei Teilaspekte sollen anhand der folgenden Fragen genauer analysiert werden:

- Wie verändert sich die Finanzierung von Unterhaltungsinhalten?

- Welche Bedeutung nehmen alternative Distributionskanäle für Produzenten ein?

Hinsichtlich der Finanzierung ist das Erlösmodell von Produktionsfirmen in Deutschland noch stark von Auftragsproduktionen geprägt, bei denen die gesamten Produktionskosten (cost plus) oder zumindest ein Teil davon (deficit financing) von den Sendern übernommen werden, die sich im Gegenzug alle Rechte sichern können (Lantzsch 2008: 98). Die Produktionsfirmen befinden sich in einer steten Abhängigkeit. Die Frage lautet deshalb, ob unter den sich wandelnden Rahmenbedingungen alternative Finanzierungsquellen an Bedeutung gewinnen werden. Insbesondere Formen programmintegrierter Werbung, wie Product Placements könnten hier eine Rolle spielen. Bloße Platzierungen von Marken könnten zur vollständigen Integration von Marken in die Handlung ausgebaut werden. Hudson und Hudson (2006: 492) sprechen von Branded Entertainment: „The integration of advertising into entertainment content, whereby brands are embedded into storylines". Eine engere Zusammenarbeit zwischen Produktionsfirmen und Werbetreibenden - ggf. unter Ausschluss der Sender - wäre eine mögliche Folge.

Das zweite Feld, in dem wir Handlungsmöglichkeiten für Produktionsfirmen sehen, betrifft die Distribution. Technische Innovationen auf Basis der Digitalisierung und Datenkompression ermöglichen den Vertrieb von Inhalten über neue Kanäle. Heute lässt sich beobachten, wie 
allem voran die Sender ihre Inhalte vermehrt online anbieten. Für TVProduktionsunternehmen stellt sich die Frage, wie im Zuge dieser Entwicklungen agiert werden soll, um auch weiterhin unternehmerisches Wachstum zu sichern. Es stellt sich die Frage, ob es eine hinreichende Zukunftsperspektive darstellt, neue Märkte mit neuen Abnehmern zu erschließen, oder in einer Vorwärtsintegration der Aufbau von Kompetenz als Distributor und Corporate Branding vielversprechender ist. Gerade in dem noch sehr unterentwickelten Bereich des Mobile-TV (Rummel 2010: 348) könnten Produktionsfirmen noch die Chance eines frühen Eintritts wahrnehmen und von den entsprechenden First-MoverVorteilen profitieren.

\section{Methode}

Der Feldzugang erfolgte über eine Kombination aus qualitativer und quantitativer Befragung. Erfasst wurde somit streng genommen nicht das tatsächliche strategische Verhalten, sondern das intendierte. Die zwei Befragungen bauten dabei aufeinander auf: Zunächst wurden Experteninterviews mit Vertretern von Produktionsfirmen und anderen involvierten Akteuren geführt. Diese telefonisch durchgeführten Leitfadeninterviews sollten die theoriegeleitete Konstruktion des standardisierten Fragebogens als zweite Stufe der Untersuchung ergänzen. So wurde sichergestellt, dass nicht nur bestehende Erkenntnisse aus der Literatur abgebildet sind, sondern auch aktuelle Entwicklungen aus der Praxis erfasst werden können. Um die Varianz abzubilden, wurden Produzenten aus verschiedenen Ländern befragt. Es wurde dabei darauf geachtet, die Fragen so offen $\mathrm{zu}$ formulieren, dass auch von unseren Erwartungen abweichende Meinungen in die Erstellung des Fragebogens einfließen konnten. Zudem wurden sämtliche relevanten Kenndaten der Branche recherchiert, um mögliche Einflussfaktoren (Marktgröße, Konzentrationsgrad, Breitbandpenetration etc.) identifizieren zu können. Das Sample konstituiert sich dabei aus Deutschland, der Schweiz, dem Vereinigten Königreich, Irland, Spanien,Kroatien, Dänemark, den Niederlanden, Schweden und Norwegen. ${ }^{1}$ Diese Auswahl deckt einerseits ein breites

1 Die Datenerhebung erfolgte in Kooperation mit Patrik Wikström und Lucia Naldi von der Jönköping International Business School. Sie haben die Kontakte in Dänemark, Schweden, Norwegen und den Niederlanden beigetragen. 
Spektrum der möglichen Einflussfaktoren ab, andererseits orientiert sie sich an der Typologie von Mediensystemen nach Hallin und Mancini (2004). Spanien und Kroatien repräsentieren dabei das „Mediterranean or Polarized Pluralist Model“", Deutschland, Dänemark, Norwegen, die Niederlande, Schweden und die Schweiz das „Northern European or Democratic Corporatist Model“ und Irland und UK das „North Atlantic Liberal Model“.

Die relevanten Produktionsfirmen wurden über die jeweiligen Branchenverbände identifiziert. Befragt wurde jeweils der Geschäftsführer oder Mitarbeiter, die in die Strategieentwicklung involviert sind. Zur Steigerung des Rücklaufs konnte auf Empfehlungsschreiben der Verbände zurückgegriffen werden, welche an die E-Mails mit der Teilnahmeaufforderung in Landessprache angehängt wurden. Der eigentliche Fragebogen war für alle Teilnehmenden derselbe und in Englisch verfasst. Ziel war eine Vollerhebung aller in Verbänden organisierten Produktionsfirmen fiktionaler und non-fiktionaler Unterhaltung in den ausgewählten Ländern. Da explizit die strategische Positionierung der Produzenten im Fokus steht, wurde auf die Befragung der anderen Akteure in der Wertschöpfungskette der audiovisuellen Unterhaltung verzichtet.

Von den insgesamt 1467 angeschriebenen Produktionsfirmen lagen schlussendlich knapp 137 ausgefüllte Fragebögen vor, was einer Rücklaufquote von knapp 10\% entspricht. Bei der Einteilung der Gruppen, die verglichen werden, wurde darauf geachtet, dass die Fallzahlen in den Gruppen ausreichend groß für statistisch relevante Aussagen blieben.

\section{Ergebnisse}

\subsection{Wahrgenommene Dynamik und Kompetenzaufbau}

Generell schätzen europäische Produktionsfirmen ihre Branche als nicht sehr dynamisch ein. Einzig Änderungen im technologischen Bereich der Produkte und Dienstleistungen werden insgesamt als eher dynamisch charakterisiert (3.57 auf einer Skala von 1, schwache bis 5, starke Branchendynamik). Signifikante Unterschiede der Ländergruppen sind nach der Unterteilung von Hallin und Mancini festzustellen ( $\mathrm{F}=4.53, \mathrm{p}<.05$, $\left.\eta^{2}=.065\right)$. Die beiden Länder mit Mediensystemen nach dem Liberal Model (UK, IE) sehen die Branche ein wenig dynamischer als die übrigen Länder. Die Unterschiede werden bei der Häufigkeit der Verände- 
rungen der Marketingaktivitäten, dem Werteverlust der Produkte, der Unberechenbarkeit der Konkurrenz sowie den technologischen Veränderung wahrgenommen. Die stärksten Unterschiede sind zwischen den demokratisch-korporatistischen und den liberalen Länder zu beobachten. Beispielhaft dafür stufen die beiden liberalen Länder die technologischen Veränderungen mit 4.00, dagegen die demokratisch-korporatistischen mit 3.24 ein $(p<.01)$. Dies könnte eine Folge der früheren Liberalisierung der Medienmärkte und Etablierung einer unabhängigen Produzentenlandschaft sein.

Die Veränderungen im technischen Bereich, im Komplexitätsgrad der Arbeitsabläufe, in der Nachfrage der Produkte und des regulatorischen Rahmens werden generell ebenso schwach wahrgenommen wie die allgemeine Branchendynamik. Einerseits wird erwartet, dass die Technik billiger wird (2.93 auf einer Skala von 1, billiger bis 7 teurer), andererseits werden die Arbeitsabläufe komplexer (4.50) und die Anforderungen an die Produktqualität leicht höher (4.11). Im Kontrast zu dem in der Wissenschaft häufig konstatierten Trend zur Deregulierung wird die Regulierung strenger werdend wahrgenommen (4.37).

Parallel zu der wahrgenommenen Branchendynamik, unterscheiden sich die beiden angelsächsischen, liberalen Länder von den anderen Ländern. Sie erwarten größere Kosteneinsparungen durch technische Veränderungen (2.42, $\left.\mathrm{F}=3.82, \mathrm{p}<.05, \eta^{2}=.056\right)$, die beiden südeuropäischen, polarisiert-pluralistischen Länder sehen hingegen nur geringfügige Einsparungsmöglichkeiten (3.31). Dass Produzenten im Vereinigten Königreich und in Irland ein größeres Kosteneinsparungspotenzial durch technische Änderungen erkennen, hängt vermutlich mit ihrer Wahrnehmung von der Branche zusammen, welche sie im Vergleich zu den anderen als dynamisch einstufen.

Um zu erfassen inwieweit die wahrgenommenen Veränderungen und Dynamiken innerhalb der Branche handlungsrelevant sind, wurden die Produktionsfirmen nach aktuellen Anpassungen an ihrem Businessmodell befragt.

Erwartungsgemäß resultiert aus der Wahrnehmung einer geringen Dynamik auch ein als schwach wahrgenommener Anpassungsdruck. Es sind kaum größere Anpassungen am Geschäftsmodell vorgenommen worden oder unmittelbar geplant. Die Schlüsselaktivitäten der Produkti- 
onsfirmen wurden insgesamt kaum verändert, dagegen stehen die beiden finanziellen Bereiche, die Kostenstruktur und die Einkommensflüsse, als Kontrast (3.28 bzw. 3.06 auf einer Skala von 1, keine Änderungen bis 5, totale Änderungen). In diesem Gebiet wurden die größten Anpassungen vorgenommen.

Aufgefallen sind grobe Tendenzen bezüglich den Ländergruppen. Produktionsfirmen in kleinen Ländern wie auch die beiden liberalen Länder waren in den letzten drei Jahren anpassungsfreudiger und haben Anpassungen an ihren Businessmodellen vorgenommen. Auch hier kann man von der Annahme ausgehen, dass durch eine unternehmerische Branchenwahrnehmung, wie dies die liberalen Länder pflegen, mehr Anpassungen vorgenommen werden und dass sie dadurch eventuell auch innovativer sind, wie dies Miller und Friesen (1982) aufgezeigt haben.

Neben dem Geschäftsmodell kann auch bei den Kompetenzen der Mitarbeitenden eine gewisse Anpassung durch veränderte Rahmenbedingungen des Markts erwartet werden. Die Produktionsfirmen wurden nach konkreten Auswirkungen gefragt, welche sich durch die Veränderung der klassischen Qualifikationen vollzogen haben.

Weder die Notwendigkeit einer Marketingabteilung (40\% Zustimmung) noch eine engere Zusammenarbeit mit Werbeagenturen (20\%) oder neue Einstellungs- und Weiterbildungsprogramme (18\%) werden als besonders erforderlich empfunden. Hingegen wird von den Mitarbeitenden erwartet, dass sie über eine größere Fülle an Kenntnissen verfügen und bereit sind sich mehr Fachkenntnisse anzueignen (74\%). Hier dürfte die durchschnittliche Größe bzw. Kleinheit der Produktionsfirmen eine Rolle spielen. In Kleinbetrieben werden vielseitige Angestellte benötigt, da das Geschäftsvolumen die Anstellung von Spezialisten nicht rechtfertigt.

Auch in Bezug auf die Mitarbeiterkompetenzen zeigen sich Unterschiede zwischen den Ländergruppen. In den angelsächsischen Ländern wurde bis anhin keine Wichtigkeit im Aufbau von Marketingabteilungen (0.21, $\left.\mathrm{F}=4.74, \mathrm{p}<.05, \eta^{2}=.068\right)$ gesehen, andererseits wurde diese Variante in den südeuropäischen Märkten teils umgesetzt (0.50). Genauso ist es der Fall bei den Einstellungs- und Weiterbildungsprogrammen, im Vereinigten Königreich und in Irland wurden die jeweiligen Programme sehr selten angepasst $\left(0.13, \mathrm{~F}=5.88, \mathrm{p}<.05, \eta^{2}=.083\right)$, dementgegen wurden 
diese Änderungen in Spanien und Kroatien teilweise vorgenommen. Denkbar ist hier ein Nachholbedarf.

Wenn Produktionen teilweise auch durch Werbung finanziert werden sollen, stellt sich die Frage, woher die notwendige Kompetenz in der Akquisition und Umsetzung kommen könnte. 25\% der Produktionsfirmen glauben keine neuen Kompetenzen zu benötigen. Es handelt sich dabei nicht um Firmen, die die Kompetenzen bereits aufgebaut haben, sondern größtenteils $(85,7 \%)$ um solche, die die Veränderung der Branche nicht oder nur schwach wahrnehmen. 33\% planen externe Kompetenz projektweise hinzuzuziehen, die übrigen $42 \%$ wollen neue Mitarbeiter einstellen bzw. erwarten, dass sich ihre Mitarbeiter in diesem Bereich weiterbilden.

\subsection{Werbefinanzierung der Produktion}

Die Produktionsfirmen wurden gebeten anzugeben, welcher Anteil ihrer Projekte in den Jahren 2007 und 2009 mindestens teilweise direkt durch die werbetreibende Wirtschaft finanziert wurde. Überdies sollte eine Schätzung für das Jahr 2011 abgegeben werden. Obwohl sich dabei zeigte, dass der Anteil von Advertiser Funded Programming (AFP) bei den Produktionen im Mittel auf relativ niedrigem Niveau startete, ist doch ein eindeutiger Trend zu erkennen: Während der Anteil 2007 durchschnittlich bei knapp 9\% lag, waren es 2009 schon 11\% und für 2011 erwarten die Befragten knapp 16\%.

Der wachsende Anteil der Werbetreibenden an der Finanzierung macht sich auch im Ablauf der Produktionen bemerkbar. Bei Produktionen ohne Werbefinanzierung sehen sich mit deutlichem Vorsprung die Produzenten selbst in der Rolle des Initiators (78\%) gefolgt von den Sendern $(16 \%)$. Andere Akteure fallen daneben kaum ins Gewicht. Für Produktionen mit Product Placement sehen Produktionsfirmen gelegentlich Werbetreibende und Werbeagenturen als Initiator $\left(\sum=20 \%\right)$, dennoch geben die Befragten auch hier an, selbst am häufigsten den Prozess einzuleiten (59\%). Eine deutlich gewichtigere Rolle spielen Werber, Media Agenturen sowie Werbeagenturen allerdings bei BrandedEntertainment-Projekten: Zusammengenommen werden diese Akteure knapp genauso oft als Initiator genannt $\left(\sum=34 \%\right)$ wie die Produzenten $(40 \%)$ und verdrängen damit die Sender vom zweiten Platz. Ein ähnli- 
ches Bild zeigt sich in Bezug auf die Green-Light Entscheidung bei der Frage, wer letztlich über die Umsetzung eines Projekts entscheidet.

Bei Sendungen ohne Werbefinanzierung sind es mehrheitlich die Sender als Auftraggeber, die das Green-Light (52\%) geben, gefolgt von den Produktionsfirmen (43\%) bei nicht komplett senderfinanzierten Projekten. Analog zur Situation bei der Initiierung eines Projekts spielen Werber und ihre Agenturen beim Product Placement eine gewisse Rolle. Weit prominenter ist ihre Rolle jedoch bei Branded-EntertainmentProduktionen: Die werbetreibende Wirtschaft hat hier mehr Entscheidungskompetenz als die Produzenten (19\%) und gibt in 21\% der Fälle das endgültige Green-Light. Auf einer siebenstufigen Skala sollten die Befragten angeben, wer primärer Empfänger von Gewinnen aus AFPs sei, sie oder die Distributoren (Sender); dabei zeigte sich eine relativ gleichmäßige Verteilung im Mittel von 4.24. Bei der Entscheidung, wie diese Mittel einzusetzen seien, weist ein Durchschnitt von 4.42 leicht in die Richtung der Produzenten.

Es lässt sich somit eine Machtverschiebung in der Branche feststellen, die den Finanzierungsanteilen folgt. Wenn werbetreibende Unternehmen die Sender als Geldgeber ersetzen, übernehmen sie auch ihre Entscheidungskompetenz. Die Produzenten bleiben in einem Abhängigkeitsverhältnis, allein der einflussnehmende Akteur ändert sich für sie.

Mit der Integration der Werbung bereits auf der Stufe Produktion verändert sich nicht nur die Finanzierung, sondern vermutlich auch die Arbeitsabläufe in der Produktion, der Schauwert einer Sendung, das Verhältnis zu anderen Stakeholdern und die Qualität der Sendungen. Die Produktionsfirmen ${ }^{2}$ geben an, dass sowohl für Product Placements als auch für Branded Entertainment zwar ein höherer Aufwand bei der Mittelbeschaffung erwartet wird, im Gegenzug jedoch auch höhere Gesamtbudgets möglich werden. Insgesamt erwarten die Produktionsfirmen eine Verbesserung ihrer Performance. Hierbei steht jedoch die finanzielle Perspektive im Vordergrund: Zwar können im Vergleich zu werbefreien Produktionen mit beiden Finanzierungsformen leicht höhere Schauwerte

2 Für diesen Aspekt wurden nur Produktionsfirmen, die bereits Erfahrung mit Product Placements oder Branded-Entertainment haben, befragt $(n=86)$. 
erzielt werden, es wird jedoch erwartet, dass die Qualität der Produktionen durch die Werbefinanzierung leicht zurückgehen wird. Im Austausch mit anderen Akteuren kann die Werbefinanzierung eine Belastung bedeuten. Eine Verschlechterung des Verhältnisses befürchten Produktionsfirmen vornehmlich zu Kreativen, erwartungsgemäß jedoch auch zu Filmförderern. In den vorab geführten Interviews wurde eine mögliche Beschränkung der Kreativen durch Vorgaben des Werbeauftraggebers allerdings als unproblematisch dargestellt. Der Vertreter einer der erfolgreichsten deutschen Produktionsfirmen formuliert es so: „Also das wird angesagt und dann wird das gemacht".

Im Folgenden soll auf Unterschiede in den oben genannten Gruppen eingegangen werden. Bei einer Einteilung der Länder nach der Typologie von Mediensystemen (Hallin \& Mancini, 2004) fällt auf, dass Produktionsfirmen aus Ländern der Kategorie „Polarized Pluralist“ die Initiative bei Produktionen ohne Werbefinanzierung deutlich stärker $(80 \%$ bei $\mathrm{p}<.1)$ bei sich selber sehen, als dies in den anderen beiden Ländergruppen der Fall ist. Bei Branded Entertainment Projekten sehen Produktionsfirmen aus UK und Irland einen größeren Einfluss der Werber bezüglich des Green-Lights $\left(\sum=44 \%\right)$ als Produktionsfirmen in anderen Ländern $(\mathrm{p}<.05)$.

Relativ deutliche Unterschiede zeigen sich bei einer Einteilung der Produktionsfirmen nach Schwerpunkt des Genres. Während FictionProduzenten etwas unter dem Gesamtdurchschnitt liegen und der Wert für Doku-Produzenten über alle Jahre recht konstant um 10\% pendelt sind Non-Fiction Produktionen offenbar stärker für Werbefinanzierung prädestiniert. 2007 hatten im Mittel 19\% der Produktionen einen Werbeanteil in der Finanzierung, 2009 bereits 26\% und für 2011 werden 31\% erwartet $(31 \%, \mathrm{p}<.1)$. Non-Fiction Branded Entertainment Produktionen werden signifikant häufiger durch die werbetreibende Wirtschaft und ihre Agenturen initiiert und zur Umsetzung freigegeben (GreenLight) als Fiction- oder Doku-Projekte. Dieses Ergebnis lässt zwei Schlüsse zu: Entweder gelten Non-Fiction Sendungen bei den Werbern als das attraktivere Umfeld, oder aber die Produzenten in diesem Bereich sind einer Werbefinanzierung ihrer Produktionen gegenüber offener eingestellt. Für beide Schlüsse gibt es Argumente: Für Werber ist es attraktiv, in einer Spielshow präsent zu sein, in der mit dem eigenen Produkt interagiert wird und bei Spielshows dürfte es weniger Probleme mit 
kreativen Autoren geben, die unabhängig von der Werbung bleiben wollen.

Häufig wird die Werbefinanzierung der Produktion nicht direkt zwischen Produzent und Werbung verhandelt, sondern über den Sender vermittelt. Dieser versucht seine Interessen zu wahren, da die Werbung innerhalb der Sendung in Konkurrenz zu den Werbeblöcken steht. Die Erlöse aus der Werbefinanzierung stehen somit nicht zwingend und häufig nicht komplett den Produzenten zur Verfügung. Im Vergleich der Genres zeigt sich, dass es Fiction- und Dokumentar-Produzenten (4.45 auf Skala von 1, Erlöse komplett beim Sender bis 7, Erlöse komplett bei Produktionsfirma) besser gelingt die Werbeerlöse für sich zu behalten als Non-Fiction-Produzenten $(4.20 \mathrm{p}<.05)$.

Unterschiede zwischen den Genres zeigen sich schließlich auch beim Kompetenzaufbau. Fiction Produzenten sehen insgesamt weniger Bedarf neue Kompetenzen aufzubauen. Unter denen, die es für nötig erachten, halten sich diejenigen die eigene Kompetenz aufbauen wollen, und diejenigen, die Kompetenz einkaufen wollen, die Waage. Unter Non-Fiction und Doku-Produzenten ist man sich relativ einig, neue Kompetenzen zu benötigen: Diese sollen vor allem im eigenen Haus angesiedelt, deutlich seltener extern eingekauft werden (60\% bzw. 33\%).

Eine Differenzierung zwischen Kino- und TV-Produzenten ergibt kaum Unterschiede. Erwartungsgemäß sehen sich Produktionsfirmen bei Kinoproduktionen stärker als Initiatoren und Entscheider, während bei TV-Produktionen den Sendern die zentrale Rolle zukommt.

Die Einteilung nach Unternehmen mit einem Anteil von mindestens 10\% Onlineproduktionen brachte aufgrund der zu geringen Fallzahl kaum signifikante Ergebnisse. Unterschiede sind jedoch bei einer Aufschlüsselung nach Unternehmensgröße in Terzile erkennbar: Dabei geben sowohl große, als auch kleine Firmen an, mehrheitlich der Profiteur der Werbefinanzierung zu sein, während mittlere Firmen angeben, dass die Erlöse mehrheitlich bei den Sender landen. Folgerichtig erwarten Unternehmen mittlerer Größe seltener, dass durch Werbefinanzierung höhere Budgets möglich sind. Hier zeichnet sich eine deutliche Beziehung zwischen Unternehmensgröße und Verhandlungsmacht ab. Große Produzenten können sich gegenüber den Sendern behaupten und ihre Interessen durchsetzen, mittlere nicht. Kleine Unternehmen, unter denen 
sich insbesondere auch viele Kinoproduzenten finden, sind wiederum genügend unabhängig von den Sendern, dass sie hier ihre Interessen wahren können. Für letztere ist das Verhältnis zu den Sendern jedoch weniger relevant als zu den Verleihern.

\subsection{Online Distribution}

In den traditionellen Distributionskanälen wird in den kommenden drei Jahren kein Wachstum mehr erwartet. Auf einer Skala von -3 (starker Rückgang) bis +3 (starkes Wachstum) erreichen das Fernsehen (-0.04) und das Kino (-0.28) aus der Sicht der Produktionsunternehmen lediglich Werte, die eine Stagnation bedeuten. Insbesondere bei der Einschätzung über die Entwicklung des Fernsehens sind hier aber signifikante Unterschiede zwischen den Ländern auszumachen $(\mathrm{F}=6.18, \mathrm{p}<.01$, $\left.\eta^{2}=.095\right)$. Während in Ländern des demokratisch-korporatistischen Modells (z.B. Deutschland, -0.66; die Schweiz, -0.69) ein deutlicher Relevanzrückgang vorausgesagt wird, sieht man in südeuropäischen Staaten des polarisiert-pluralistischen Modells (Spanien, 0.53; Kroatien, 0.65) auf mittelfristige Sicht sogar eine positive Entwicklung. Die Werte für die ,liberalen` Länder liegen im Nullbereich. Für neue Vertriebskanäle wird erwartungsgemäß ein relativ starkes Wachstum prognostiziert, wobei man für Mobile (1.44) über alle Gruppen hinweg etwas skeptischer ist als für Online (1.82). Auf dem Onlinemarkt wird dabei der Wettbewerb insgesamt als eher schärfer bezeichnet als auf den traditionellen Märkten TV und Kino. Je dynamischer die eigene Branche wahrgenommen wird, desto eher wird auch der Wettbewerb im Onlinebereich als scharf eingestuft $(\beta=.18, p<.05)$, obwohl weniger als ein Fünftel der befragten Produktionsunternehmen tatsächlich nennenswert für diesen Distributionskanal produzieren (24 von 137 mit mindestens 10\% des Gesamtoutputs für Online).

Der Onlinemarkt wird demnach wachsen, jedoch ist er umkämpft. Die Produktionsfirmen sehen sich dabei nicht als die Nutznießer der Entwicklung. An Bedeutung gewinnen, sei es als Konkurrenten oder Geschäftspartner, werden nach ihrer Einschätzung vor allem Plattformbetreiber im Internet, die das Nutzerinteresse auf sich fokussieren können (Google, Apple, etc.). Ebenfalls an Bedeutung gewinnen könnten Netzwerkbetreiber, die die technische Infrastruktur bereitstellen, sowie im geringeren Masse die Nutzer selbst, im Rahmen von user generated content (UGC). Insbesondere kleine Produktionsunternehmen sehen im 
UGC ein signifikant höheres Potenzial zur Zusammenarbeit, beziehungsweise direkten Konkurrenz als große Unternehmen ( $\mathrm{F}=4.59$, $\left.\mathrm{p}<.05, \eta^{2}=.07\right)$. Auf den letzten Rängen liegen gemäß den Produzentenprognosen die Medienunternehmen (Sender, Verlagshäuser etc.), sowie andere Produktionshäuser. Weitgehend analog zur Bedeutung der Akteure ist die erwartete Rangreihe in Bezug auf den Erlösanteil am Onlinegeschäft. Auch hier belegen Internet- und Telekommunikationsfirmen die vordersten Ränge und greifen folglich die größten Gewinne ab. TVSender, die Werbetreibenden, sowie ihre Werbeagenturen bilden die Mittelgruppe. Für Produktionsunternehmen und die Nutzer selbst (UCG) bleiben somit auf dem Onlinemarkt im Vergleich mit anderen Akteuren keine Möglichkeiten mehr, sich an den Erlösen entscheidend zu beteiligen. Die Produzenten erwarten somit, dass die Entwicklung im audiovisuellen Bereich weitgehend jener im Musikbereich und im Printjournalismus gleichen wird. Signifikante Unterschiede zeigen sich zwischen den Ländergruppen ( $\left.F=5.09, \mathrm{p}<.01, \eta^{2}=.07\right)$ : In den südeuropäischen Märkten (Spanien und Kroatien) glauben Produktionsunternehmen im Onlinegeschäft ähnlich hohe Erlösanteile wie die Sender realisieren zu können, während in den, liberalen' Staaten UK und Irland auf die eigene Akteursgruppe bezogen der größte Pessimismus herrscht. Der entsprechende Wert für die Länder des demokratischkorporatistischen Modells liegt dazwischen, geht dabei eher in die Richtung der letzteren Gruppe, allerdings weniger dramatisch.

Sofern eine Vermarktung im Internet erfolgen soll, halten es die Produzenten für relativ wichtig, Änderungen am Produkt selbst vorzunehmen. In diesem Zusammenhang geht es vor allem darum, das jeweilige Gesamtbudget und die Länge einer einzelnen Episode anzupassen. Die Bedeutung von ersterem wird in großen Ländern, in denen die Produktionsmärkte von einer höheren Vielfalt und Segmentierung gekennzeichnet sind, tendenziell höher eingeschätzt als in kleinen Staaten, wo ein tieferer Wettbewerbsdruck vermutet werden könnte. Dieselbe Differenz ist auch zwischen Produzenten von fiktionalen und non-fiktionalen Inhalten zu beobachten. Letztere sehen im Onlinebereich einen signifikant höheren Zwang zur Budgetanpassung $\left(F=5.37, p<.01, \eta^{2}=.08\right)$. Aus ihrer Perspektive muss auch die Episodenlänge eher angepasst werden als dies bei Fiction-Produzenten der Fall ist $\left(\mathrm{F}=4.07, \mathrm{p}<.05, \eta^{2}=.06\right)$. Als Indiz dafür, dass sich die Länge der Unterhaltungsformate im Internet tatsächlich ändern muss, um eine erfolgreiche Vermarktung wahrscheinlicher zu 
machen, lässt sich im Urteil von bereits erfahrenen Online-Produzenten ablesen: Die Produktionsunternehmen, welche bereits mindestens zehn Prozent ihres Outputs für das Internet produzieren, teilen eine solche Einschätzung eher, als jene ohne bedeutende Onlineproduktionen $\left(\mathrm{F}=5.79, \mathrm{p}<.05, \eta^{2}=.04\right)$.

Nachdem geklärt wurde, dass das Internet als Distributionskanal ein hohes Potenzial hat, Produktionsunternehmen aber gemäß ihrer heutigen Einschätzungen kaum Nutzen daraus ziehen können - obwohl sie mit ihrer Produktionskompetenz am ehesten wüssten, wie die Produkte für den neuen Markt zu optimieren wären - stellt sich die Frage, ob die Vorwärtsintegration der Distribution als strategische Optionen aussichtsreich wäre, um so die eigenen Produktionskompetenz besser kapitalisieren zu können. Die befragten Produktionsunternehmen selbst sind diesbezüglich eher pessimistisch. Bestrebungen, selbst produzierte Programme auch selbst zu vertreiben, werden nur als mittelmäßig attraktiv eingeschätzt. Ein klarer Unterschied besteht in dieser Frage aber zwischen den Ländergruppen: Während zentraleuropäische und skandinavische Länder des Democratic-Corporatist-Modells zu diesem Aspekt keinen Handlungsbedarf sehen, sind in polarisiert-pluralistischen und liberalen Staaten tendenziell höhere Werte zu verzeichnen. Wird hingegen nach der Kompatibilität mit dem bisherigen Geschäftsmodell gefragt, so teilen die Befragten über alle Gruppen hinweg die Meinung, dass eher keine Synergien vorhanden sind und Produktionsunternehmen dadurch in einen für sie völlig neuartigen Geschäftsbereich einsteigen müssten. Eine Vielzahl von Kompetenzen werden zu diesem Zweck als wichtig eingestuft: Verkaufskompetenz, Branding, technische Aspekte, Marktmacht bzw. eine breite Kundenbasis, sowie Kosteneffizienz. Bei den Befragten überwiegt die Meinung, dass ein Produktionshaus diese Kompetenzen nicht aufbauen kann und soll. Die Resultate deuten also darauf hin, dass auch im Onlinebereich die klassische Rolle des Produzenten angenommen wird.

\section{$6 \quad$ Fazit und Limitierungen}

Unsere Ergebnisse bestätigen den auf Basis von Interviewstudien (Przybylski 2010; Rott/ Zabel, 2009) gewonnen Eindruck, dass Unterhaltungsproduzenten nicht daran interessiert sind die Veränderungen in der Branche als Anlass zu nehmen eine Vorwärtsintegration der Onlinedistribution zu versuchen. Die Produktionsfirmen sehen kaum Sy- 
nergien mit dem bisherigen Geschäft, sondern eine Notwendigkeit viele neue Kompetenzen aufzubauen. Da man sich gegenüber Sendern mit etablierten Brands und Online-Plattformen mit großer Rezipientennachfrage in einer Außenseiterposition sieht, ist die Bereitschaft die notwendigen Kompetenzen aufzubauen entsprechend gering. Allerdings müssen hier Unterschiede zwischen verschiedenen TV-Märkten konstatiert werden. In Märkten mit einer längeren Tradition von unabhängigen Produktionsfirmen haben sich diese im Vergleich zu den Sendern eine stärkere Position erarbeitet und zeigen auf Basis dieser relativ größeren Marktmacht heraus auch mehr Interesse an einer Vorwärtsintegration. Dennoch: Produktionsfirmen sehen sich klar im b2b-Geschäft und haben wenig Interesse Consumerbrands aufzubauen. Das bedeutet jedoch nicht, dass Produktionsfirmen gar nicht auf Veränderungen in den Rahmenbedingungen reagieren würden. Es zeigt sich ein wachsender Anteil von Werbefinanzierung bereits in der Produktion und eine große Bereitschaft im Bereich der Werbegeldakquisition Kompetenzen aufzubauen oder wenigstens fallweise einzukaufen. Die Produktionsfirmen machen sich jedoch keine Illusion, dass eine Werbefinanzierung als Emanzipation von den Sendern zu verstehen ist. Vielmehr handelt es sich um eine passive Reaktion in der sich mit dem Finanzierungsbeitrag auch der Einfluss von Sendern zu Werbetreibenden verschiebt. Unterschiede zeigen sich hier weniger in Bezug auf die nationalen Märkte oder Mediensystemtypen, sondern vielmehr in Bezug auf das produzierte Programm. Bei Dokumentationen als Informationsinhalt sind die Möglichkeiten einer Werbefinanzierung beschränkt, und auch im fiktionalen Bereich gibt es Vorbehalte wegen einer allfälligen Beschränkung der künstlerischen Freiheit. Im Non-Fiction Bereich lassen die Ergebnisse in den kommenden Jahren jedoch eine große Verbreitung von werbefinanzierten Produktionen erwarten.

Bezogen auf die Wertschöpfungskette, integrieren Produktionsfirmen also lieber die parallele Wertschöpfungskette der Werbung, als dass sie eine Vorwärtsintegration wagen. Die Kernkompetenz der Produktion steht weiterhin im Vordergrund und das vorhandene Vertriebstalent wird auf neue Geldgeber ausgeweitet.

Einschränkungen der gefundenen Ergebnisse müssen selbstverständlich in Bezug auf die Ausschöpfungsquote gemacht werden. Allerdings zeigt ein Blick in die Teilnehmerliste, dass die wichtigsten Produktionsfirmen in den jeweiligen Ländern meist bereitwillig an der Umfrage teilgenom- 
men haben. Daneben finden sich im Sample viele kleine Firmen, eine eher schlechte Ausschöpfung gibt es bei Unternehmen mittlerer Größe. Zwischen den Ländern gibt es Unterschiede nicht nur bezüglich der Ausschöpfung, sondern bereits bezüglich des Organisierungsgrads der Branche, also des Anteils der Produktionsfirmen, die über die Mitgliederlisten der Verbände erreicht werden können. Denkbar wäre hier eine systematische Unterschätzung der Bedeutung von Werbefinanzierung und Online Distribution, da sich Unternehmen, die in diese Bereiche expandieren ggf. nicht gut von den klassischen Verbänden vertreten fühlen und entsprechend seltener Mitglied sind.

Das Problem von sozialer Erwünschtheit bei Fragen, welche die Trennung von Werbung und Programm tangieren scheint in Anbetracht der Aussagen der Branchenvertreter in den Interviews dagegen ein rein akademisches Konstrukt. Werbefinanzierung der Produktion ist legal, absolut akzeptiert und wird im Unterhaltungskontext ohnehin als unproblematisch gesehen. Eine Verfälschung der Aussagen ist eher denkbar im Sinne der Selbstdarstellung der Produktionsfirmen. Sie legen Wert auf ihre kreative Expertise und könnten somit versucht sein in ihren Antworten diese Perspektive übermäßig zu betonen. Verzerrungen durch die Selbstdarstellung ließe sich nur durch eine umfassende Befragung aller Akteure im Wertschöpfungsnetzwerk realisieren, um so jeweils Binnenund Außenperspektive abgleichen zu können.

\section{Literatur}

Altmeppen, Klaus-Dieter (2008): Wer macht was? Organisationale Handlungsfelder in der TV-Contentproduktion. S. 30-53 in: Siegert Gabriele/ von Rimscha, M. Bjørn (Hrsg.): Zur Ökonomie der Unterhaltungsproduktion. Köln: Herbert von Halem.

Ansoff, Harry I. (1968): Corporate strategy: An analytic approach to business policy for growth and expansion. Harmondsworth: Penguin.

Chaston, Ian (2008): Small creative industry firms: A development dilemma? S. 819-831 in: Management Decision. 46/6.

Doyle, Gilian (2002): Understanding media economics. London: Sage.

Fröblich, Kerstin (2010): Innovationssysteme der TV-Unterhaltungsproduktion: Komparative Analyse der sektoralen Innovationsbedingungen Deutschlands und Großbritanniens. Wiesbaden: VS Verlag.

Hallin, Daniel C./ Mancini, Paolo (2004): Comparing media systems: Three models of media and politics. Communication, society and politics. Cambridge: Cambridge University Press.

Hudson, Simon/Hudson, David (2006): Branded entertainment: A new advertising technique or product placement in disguise? S. 489-504 in: Journal of Marketing Management. $22 / 5 \& 6$. 
Lantzsch, Katja (2008): Der internationale Fernsehformathandel: Akteure, Strategien, Strukturen, Organisationsformen. Wiesbaden: VS Verlag.

Miller, Danny/ Friesen, Peter H. (1982): Innovation in conservative and entrepreneurial firms: Two models of strategic momentum. S. 1-25 in: Strategic Management Journal, $3 / 1$.

Pätrold, Ulrich/Röper, Horst (2003): Fernsehproduktionsvolumen 1998 bis 2000. Fortschreibung der Formatt-Studie über Konzentration und regionale Schwerpunkte der Auftragsproduktionsbranche. S. 24-34 in: Media Perspektiven. 2003/1.

Picard, Robert G. (2004): Environmental and market changes driving strategic planning in media firms. S. 1-17 in Picard, Robert G. (Hrsg.): Strategic responses to media market changes. Jönköping: Jönköping International Business School.

Praybylski, Pamela (2010): Heute Partner - Morgen Konkurrenten?: Strategien, Konzepte und Interaktionen von Fernsehunternehmen auf dem neuen Bewegtbild-Markt. Wiesbaden: VS Verlag.

Rott, Armin/ Zabel, Christian (2009): Marktentwicklung als strategische Option für TV-

Produktionsunternehmen: Perspektiven, Probleme und empirische Evidenz. S. 93-106 in: Krone, Jan (Hrsg.): Fernsehen im Wandel. Mobile TV \& IPTV in Deutschland und Österreich. Baden-Baden: Nomos.

Rummel, Rene (2010): MobileTV: Kurzer Bericht aus der Praxis und Plädoyer an die Wissenschaft. S. 345- 351 in: Lantzsch, Katja/ Altmeppen Klaus-Dieter/Will, Andreas (Hrsg.): Handbuch Unterhaltungsproduktion. Beschaffung und Produktion von Fernsehunterhaltung. Wiesbaden: VS Verlag.

Sydow, Jörg/ Windeler, Arnold (2004): Projektnetzwerke: Management von (mehr als) temporären Systemen. S. 37-54 in: Sydow, Jörg/ Windeler Arnold (Hrsg.): Organisation der Content-Produktion. Wiesbaden: VS Verlag.

Tunstall, Jeremy (1993): Television producers. London: Routledge.

von Rimscha, M. Bjorn (2008): Risikomanagement in der Produktion und Entwicklung audiovisueller fiktionaler Unterhaltung. S. 178-203 in: Siegert, Gabriele/von Rimscha, M. Bjørn (Hrsg.): Zur Ökonomie der Unterhaltungsproduktion. Köln: Herbert von Halem.

Wirts, Bernd W. (2009): Medien- und Internetmanagement. Wiesbaden: Gabler Verlag.

Zabel, Christian (2008): The show must go online: Produktion für das Online-Fernsehen. Köln: HMR International. 